\title{
Upscaling of multi-beam x-ray ptychography for efficient $x$-ray microscopy with high resolution and large field of view
}

Cite as: Appl. Phys. Lett. 118, 171102 (2021); https://doi.org/10.1063/5.0045571

Submitted: 27 January 2021 . Accepted: 09 April 2021 . Published Online: 27 April 2021

(D) Felix Wittwer, (D) Mikhail Lyubomirskiy, (iD) Frieder Koch, (D) Maik Kahnt, (D) Martin Seyrich, (D) Jan Garrevoet, Christian David, and (iD Christian G. Schroer

ARTICLES YOU MAY BE INTERESTED IN

Negative effect of cations out-diffusion and auto-doping on switching mechanisms of transparent memristor devices employing ZnO/ITO heterostructure

Applied Physics Letters 118, 173502 (2021); https://doi.org/10.1063/5.0047036

Carbon-dioxide absorption spectroscopy with solar photon counting and integrated lithium niobate micro-ring resonator

Applied Physics Letters 118, 171103 (2021); https://doi.org/10.1063/5.0045869

Extreme ultraviolet photoemission of a tin-based photoresist

Applied Physics Letters 118, 171903 (2021); https://doi.org/10.1063/5.0047269

Challenge us.

What are your needs for periodic signal detection?

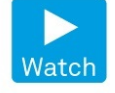

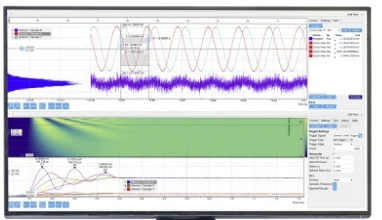

0
Zurich - Instruments 


\title{
Upscaling of multi-beam x-ray ptychography for efficient $x$-ray microscopy with high resolution and large field of view
}

Cite as: Appl. Phys. Lett. 118, 171102 (2021); doi: 10.1063/5.0045571

Submitted: 27 January 2021 - Accepted: 9 April 2021 .

Published Online: 27 April 2021

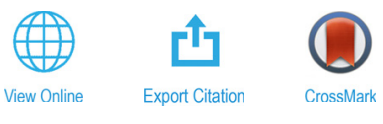

Felix Wittwer, ${ }^{1,2, a)}$ (D) Mikhail Lyubomirskiy, ${ }^{1}$ (D) Frieder Koch, ${ }^{3}$ (D) Maik Kahnt, ${ }^{1,2,4}$ (iD Martin Seyrich, ${ }^{1,2}$ (D) Jan Garrevoet, (iD Christian David, ${ }^{3}$ and Christian G. Schroer ${ }^{1,2}$ (iD)

\author{
AFFILIATIONS \\ 'Deutsches Elektronen-Synchrotron (DESY), Notkestr. 85, 22607 Hamburg, Germany \\ ${ }^{2}$ Department Physik, Universität Hamburg, Luruper Chaussee 149, 22761 Hamburg, Germany \\ ${ }^{3}$ Paul Scherrer Institut (PSI), Forschungsstr. 111, 5232 Villigen, Switzerland \\ ${ }^{4}$ MAX IV Laboratory, Lund University, Box 118, 22100 Lund, Sweden
}

${ }^{a)}$ Author to whom correspondence should be addressed: wittwer@xray-lens.de

\begin{abstract}
Nondestructive imaging with both a large field of view and a high spatial resolution is crucial to understand complex materials and processes in science and technology. X-ray ptychography can provide highest spatial resolution but is limited in the field of view by the acquisition time and coherent flux at modern x-ray sources. By multi-beam ptychography, the sample can be imaged in parallel by several spatially separated and mutually incoherent beams. We have implemented this method using 3D nanoprinted x-ray optics to create tailor-made x-ray multi-beam arrays. The use of $3 \mathrm{D}$ printing allows us to create focusing optics with a minimum of nonfunctional support structures. In this way, large sample areas can be efficiently scanned in parallel with up to six illuminating beams.
\end{abstract}

Published under license by AIP Publishing. https://doi.org/10.1063/5.0045571

$\mathrm{X}$-ray microscopy is an indispensable tool in many scientific fields due to its ability to image chemical and physical processes under realistic conditions. A key technique for high-resolution x-ray microscopy is scanning coherent diffractive imaging, also known as ptychography. It allows us to solve various scientific questions concerning, e.g., biology, ${ }^{2}$ catalysis, ${ }^{3}$ or nanotechnology. ${ }^{4}$ Many of these questions involve searching for a needle in a haystack, i.e., finding a small detail in a large object, for example, finding a local defect in a microchip. Searching for a small detail requires the imaging of a large field of view with high resolution. However, for scanning techniques such as ptychography, scanning large areas with high resolution requires long scan times. Demonstration experiments have been able to image on the order of 100 mega pixels per hour at a resolution of $40 \mathrm{~nm} .^{5}$ To push this to the level of imaging tera pixels at a resolution of $10 \mathrm{~nm}$, as demanded, e.g., by the RAVEN program of IARPA, ${ }^{6}$ would increase the scan time by years. ${ }^{7}$ Here, we propose a multi-beam scanning approach that will significantly speed-up scan times. As conventional ptychography requires a coherent beam, the scanning time is ultimately limited by the available coherent photon flux. Multi-beam ptychography can accept a larger incoherent part of the incoming beam, allowing us to scan large sample areas in parallel.

In multi-beam ptychography, multiple independent beams simultaneously scan different parts of the sample, allowing rapid scanning of large samples without sacrificing spatial resolution. ${ }^{8}$ This can be achieved because each beam only needs to be coherent with itself and can be incoherent to all other beams. Multiple groups have demonstrated multi-beam x-ray ptychography using pinholes ${ }^{9}$ or Fresnel zone plates ${ }^{10}$ to create the beams. However, these schemes leave large parts of the incoming beam unused, as both the pinholes and the zone plates require a significant dead area between the individual optics.

In this Letter, we report the use of additive $3 \mathrm{D}$ nanoprinting ${ }^{11}$ to manufacture multi-beam $\mathrm{x}$-ray optics. Taking advantage of this technology, ${ }^{12,13}$ we have fabricated lens towers that achieve sub-micron focusing of hard $\mathrm{x}$-rays. ${ }^{14}$ Owing to the geometrical freedom of $3 \mathrm{D}$ printing, we can create multiple beams by printing multiple lens towers in proximity to each other. In the current work, we demonstrate a flexible multi-beam setup for $\mathrm{x}$-rays that allows us to tailor the beam 
configuration and beam separation and is ideally suited to scan large areas quickly.

We performed the experiment at the microprobe end-station of beamline P06 ${ }^{15}$ of PETRA III at DESY in Hamburg at a photon energy of $7 \mathrm{keV}$. The experimental setup is illustrated in Fig. 1(a). We used an array of $3 \mathrm{D}$-printed refractive lenses ${ }^{14}$ to create multiple parallel beams. Two lenses are sketched in Fig. 1(b), one intact and the other cut open to show the interior. A single lens has a square footprint of $45 \mu \mathrm{m} \times 45 \mu \mathrm{m}$ and a length of $600 \mu \mathrm{m}$ and contains four parabolic surfaces with a curvature of $2.5 \mu \mathrm{m}$ to focus $\mathrm{x}$-rays. The refractive index decrement $\delta$ of the printed polymer at $7 \mathrm{keV}$ is $5.54 \times 10^{-6}$. Each lens, thus, has a focal distance of $113 \mathrm{~mm}$ (at $7 \mathrm{keV}$ ) and a diffraction-limited spot size of $335 \mathrm{~nm}$. The lenses are placed in a $47 \mu \mathrm{m}$ raster to avoid contact between neighboring lenses and to minimize manufacturing errors. This $2 \mu \mathrm{m}$ gap between the lenses leaves up to $90 \%\left(=45^{2} \mu \mathrm{m}^{2} / 47^{2} \mu \mathrm{m}^{2}\right)$ of the incoming photon beam for measurements.

Using the slits in front of the lens array, we selectively illuminate lenses to create different multi-beam configurations. Background scattering from the optics is reduced by placing a tailor-made pinhole array between the lenses and the sample. This array of $30 \mu \mathrm{m}$ pinholes is made by laser ablation from a $100 \mu \mathrm{m}$ thick platinum foil. Some of the different possible beam arrangements are shown in Fig. 1(c). This

(a)

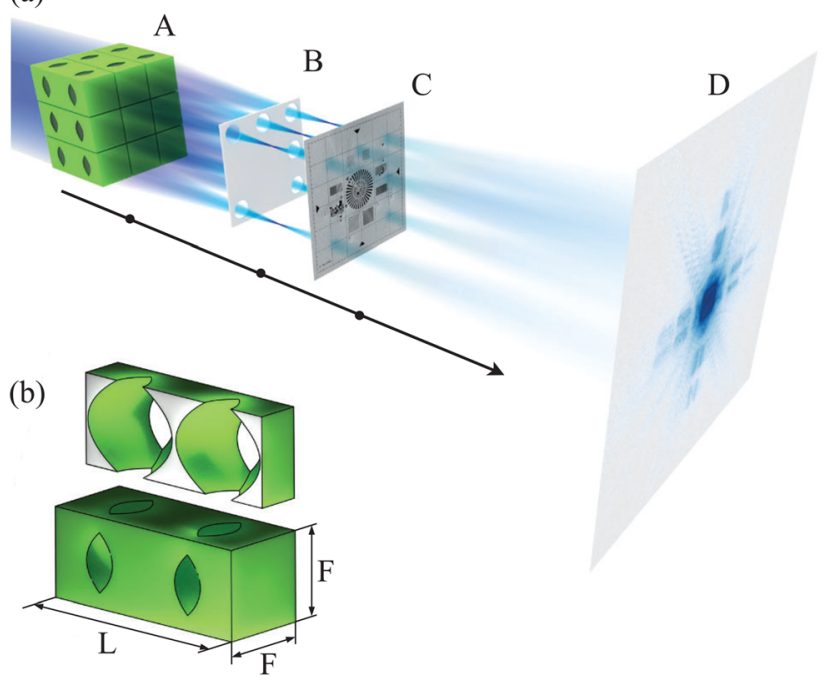

(c)

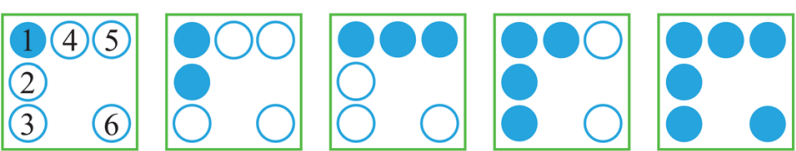

FIG. 1. (a) Experimental setup with the lens array (A) focusing multiple beams to a plane $20 \mathrm{~mm}$ upstream of the sample (C). Six of the beams are selected by the pinhole array (B) and scatter off the sample. The resulting diffraction patterns all overlap on the detector (D). (b) Not-to-scale sketches of the lens design, the top one is cut open to show the four parabolic surfaces. Each lens has a length $L$ of $600 \mu \mathrm{m}$ and a foot print $\mathrm{F}$ of $45 \mu \mathrm{m} \times 45 \mu \mathrm{m}$. The openings are necessary to drain the unused precursor after the printing. (c) Five exemplary beam configurations; the pinhole array in combination with the upstream slits allows us to create one, two, three, four, or six beams. particular arrangement of pinholes was chosen for this first experiment to test different beam separations of $47 \mu \mathrm{m}, 94 \mu \mathrm{m}$, and combinations of both. A three by three beam array can be implemented for large-field-of-view imaging in future applications.

The sample is placed $20 \mathrm{~mm}$ behind the focal plane of the lenses. At the sample position, each beam has a diameter of $7.5 \mu \mathrm{m}$. As test samples we chose two NTT-AT (XRESO-50HC) Siemens star resolution charts that feature $50 \mathrm{~nm}$ structures. We use one test chart in the two-beam measurement and the other one for the six-beam case. After the beam is scattered off the sample, the diffraction patterns are detected using a single-photon counting, custom made in-vacuum Eiger $4 \mathrm{M}$ detector (pixel size $75 \mu \mathrm{m}$ ) ${ }^{16}$ The detector is mounted at the end of the evacuated flight tube in order to eliminate absorption and scattering in air. ${ }^{17}$ The distance $z$ from the sample to the detector is $8050 \mathrm{~mm}$.

In the first measurement, the most simple multi-beam setup is used comprising two beams. We select two vertically neighboring lenses [pinhole 1 and 2 in Fig. 1(c)], to create two beams with a separation of $47 \mu \mathrm{m}$. The test sample is scanned in a grid of $16 \times 31=496$ positions in $2 \mu \mathrm{m}$ steps with $1 \mathrm{~s}$ exposure per point. To avoid scan grid artifacts, ${ }^{18}$ each point is randomly offset horizontally and vertically up to $\pm 0.25 \mu \mathrm{m}$. The scan areas of the two beams overlap on a $30 \mu \mathrm{m} \times 13 \mu \mathrm{m}(\mathrm{h} \times \mathrm{v})$ wide area.

We reconstruct the two-beam data set twice, first with single beam algorithms and then a second time with multi-beam algorithms. For both reconstructions, the Eiger images are cropped to $512 \times 512$ pixels, resulting in a reconstructed pixel size of $37.1 \mathrm{~nm}$. For the single beam reconstruction, we use a combination of 500 iterations of the standard ePIE algorithm and 500 iterations of a parallel update, using the coupled object and probe equations described by Thibault et al.. ${ }^{19}$ In contrast to ePIE, this update applies the changes from all diffraction patterns in parallel. The object and probe updates are, respectively,

$$
\begin{gathered}
O_{l+1}(\mathbf{r})=O_{l}(\mathbf{r})+\alpha \cdot \frac{\sum_{j} P_{l}^{*}\left(\mathbf{r}-\mathbf{r}_{j}\right) \phi_{j}(\mathbf{r})}{\sum_{j}\left|P_{l}\left(\mathbf{r}-\mathbf{r}_{j}\right)\right|^{2}+\epsilon}, \\
P_{l+1}(\mathbf{r})=P_{l}(\mathbf{r})+\beta \cdot \frac{\sum_{j} O_{l}^{*}\left(\mathbf{r}+\mathbf{r}_{j}\right) \phi_{j}\left(\mathbf{r}+\mathbf{r}_{j}\right)}{\sum_{j}\left|O_{l}\left(\mathbf{r}+\mathbf{r}_{j}\right)\right|^{2}+\epsilon} .
\end{gathered}
$$

Here, $\epsilon$ is a small regularization constant $\left(\sim 10^{-3}\right)$ and $\phi_{j}(\mathbf{r})$ $=\psi_{j}^{\prime}(\mathbf{r})-\psi_{j}(\mathbf{r})$ is the difference between the updated and the previous exit wavefront.

As the sample scanning stage did not reach the dialed positions with the necessary precision, a brute-force position refinement was used to improve the reconstruction. ${ }^{20}$ The refined positions are used for all the following reconstructions of this scan.

In this single beam reconstruction, the reconstructed probe is a combination of the two beams. Similarly, the reconstructed object, shown in Fig. 2(b), is a combination of the two scanned regions. These combinations result in some features appearing twice in the reconstruction. Measuring the distance between the two copies of the same feature provides a more accurate estimate of the distance between the two beams. Here, the center of the Siemens star repeats after $45.5 \mu \mathrm{m}$. This slight deviation from the design value of $47 \mu \mathrm{m}$ can be caused by distortions in the wave front or manufacturing errors in the optics.

For the multi-beam reconstruction, we use a modified version of the incoherent multiplex algorithm from the study by Batey et al. ${ }^{21}$ 


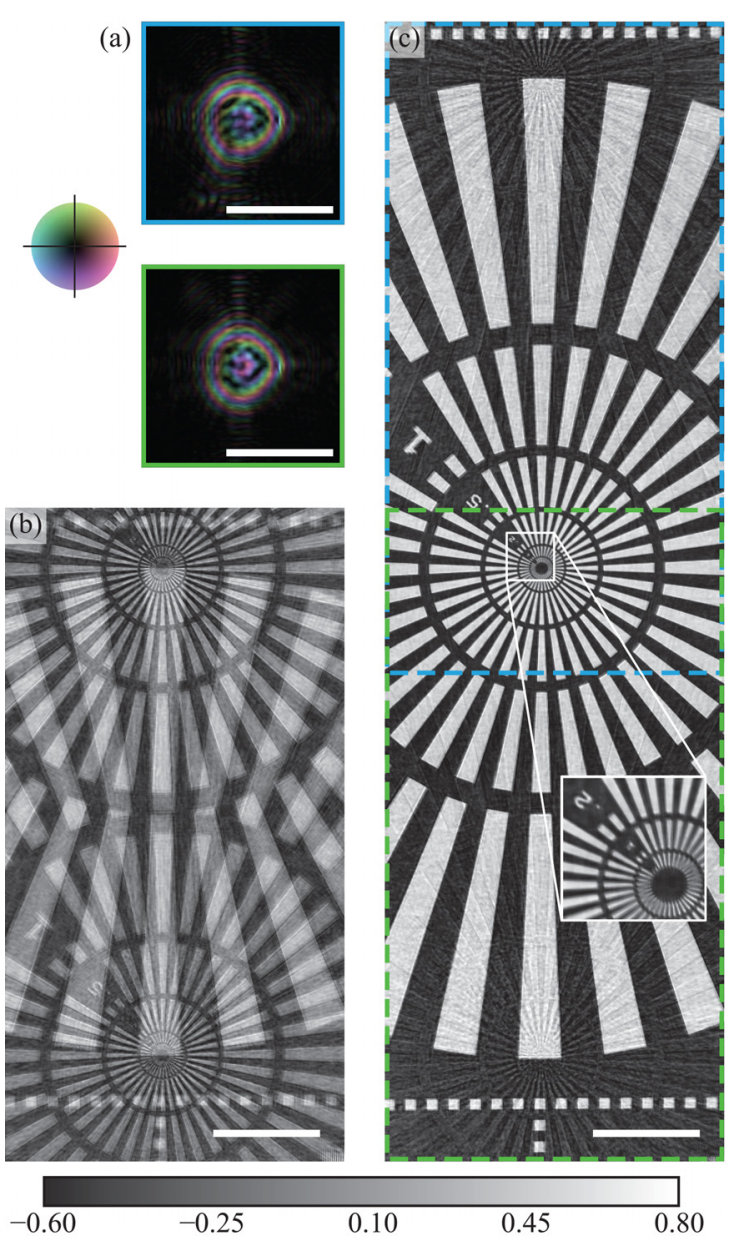

FIG. 2. Reconstructions of the two-beam measurement. (a) and (c) use the multibeam algorithm, and (b) uses the single beam ePIE algorithm. (a) shows the two probes in a complex colorscale. (b) shows the phase shift of the Siemens star reconstructed with ePIE. (c) shows the multi-beam reconstruction of the Siemens star, with the green rectangle indicating the area scanned by the first probe and the blue rectangle indicating the area scanned by the second probe. The zoom-in shows an enlarged view of the center of the Siemens star. All white scale bars represent $10 \mu \mathrm{m}$. The Siemens stars in (b) and (c) share the same colorscale.

This algorithm was designed to scan a sample at multiple wavelengths in a single measurement. Here, all probes have the same wavelength but scan different parts of the sample. To this end, each beam has a different optical axis and beam center on the detector. By initializing each beam in the sample plane with a suitable phase wedge factor, their footprint on the detector can be shifted individually. Adapting the multiplex algorithm for multi-beam ptychography is straightforward, and deriving a corresponding parallel multi-beam update is similarly possible.

With the modified multiplex algorithm, the two-beam data set is reconstructed for 500 iterations, followed by a further 500 iterations using the multi-beam parallel update.

The reconstructed probes are shown in Fig. 2(a). Although the nano-printing process is highly repeatable, the two probes are not identical and show small differences. With the separation of the probes, the reconstruction is able to separate the two scan areas and reconstruct the full length of the Siemens star [see Fig. 2(c)]. In the central region, which was illuminated by both beams during the scan, very weak artifacts are visible. Outside this shared band, where only one of the two beams illuminated the sample, the artifacts are stronger, most prominently at the top and bottom of the reconstruction. These ghost artifacts arise because the two probes are partially coherent and interfere on the detector, creating interference stripes in the diffraction patterns. The stripes can be suppressed to make the beams effectively incoherent by choosing a beam separation that is an integer multiple of

$$
D=\lambda z / p,
$$

where $\lambda$ is the wavelength of the $\mathrm{x}$-rays, $z$ is the distance between the sample and the detector plane, and $p$ is the detector pixel size. Here, the characteristic beam separation $D$ is $19 \mu \mathrm{m}$, which means that the nominal beam separation of $47 \mu \mathrm{m}$ corresponds to $2.5 \mathrm{D}$, halfway between the optimal values of $2 D$ or $3 D$. This suboptimal distance implies that the interference is only partially suppressed, here to about $15 \%$ of its maximum value. Correspondingly, the reconstruction algorithm cannot fully separate the two beams, giving rise to the aforementioned artifacts in the reconstructed sample. This problem is amplified by the similarity of the probes, which adds difficulty to the separation.

To estimate the resolution, we split the data set into half and reconstruct both halves separately. We align the two reconstructions ${ }^{22}$ and calculate the resolution with the Fourier ring correlation (FRC) and the half-bit criterion to $47 \mathrm{~nm}$. The finest details of the Siemens star, the $50 \mathrm{~nm}$ spikes in the center, are faithfully reconstructed, matching the FRC result.

Scanning this object with the same fluence using a single beam would have taken 1.8 times longer than this two-beam scan. The improvement is smaller than the number of beams due to the shared band that was scanned by both beams. Using three lenses to create three beams, an even larger area could be scanned in the same time. The gain in this case compared to a single beam would be 2.6.

Even greater gains are possible with more parallel beams. To this end, the slits in front of the optics were opened to fully illuminate the lens array, creating six parallel beams behind the pinhole array as shown in Fig. 1(c) on the far right. The nominal separation between the outermost beams is $94 \mu \mathrm{m}$, twice the distance between two individual lenses. In a second ptychographic experiment, a damaged Siemens star was scanned across the beams in a raster of $120 \times 120$ steps of $1 \mu \mathrm{m}$, so that together, the multi-beams scan an area of $(120 \mu \mathrm{m}+94 \mu \mathrm{m})^{2}$. Each scan point was randomly offset in the horizontal and vertical direction up to $\pm 0.25 \mu \mathrm{m}$, and the detector was exposed each time for $0.3 \mathrm{~s}$. As the scan range is larger than the maximum beam separation, the individual scan areas of each beam overlap in the center of the Siemens star.

For the reconstruction, we combine 1000 iterations of the multibeam adapted sPIE algorithm ${ }^{23,24}$ with a $2 \times 2$ upsampling and then the corresponding parallel update for a further 1000 iterations. The use of sPIE allows us to reconstruct a larger field of view for each probe [compare Figs. 2(a) and 3(a6)]. As reconstructing six beams is more challenging than one or two beams, the weak aberrations of the lenses have more influence on the reconstruction quality and make enlarging the field of view necessary. We also use a brute-force position refinement. After the two-beam scan and similar measurements, all showed 

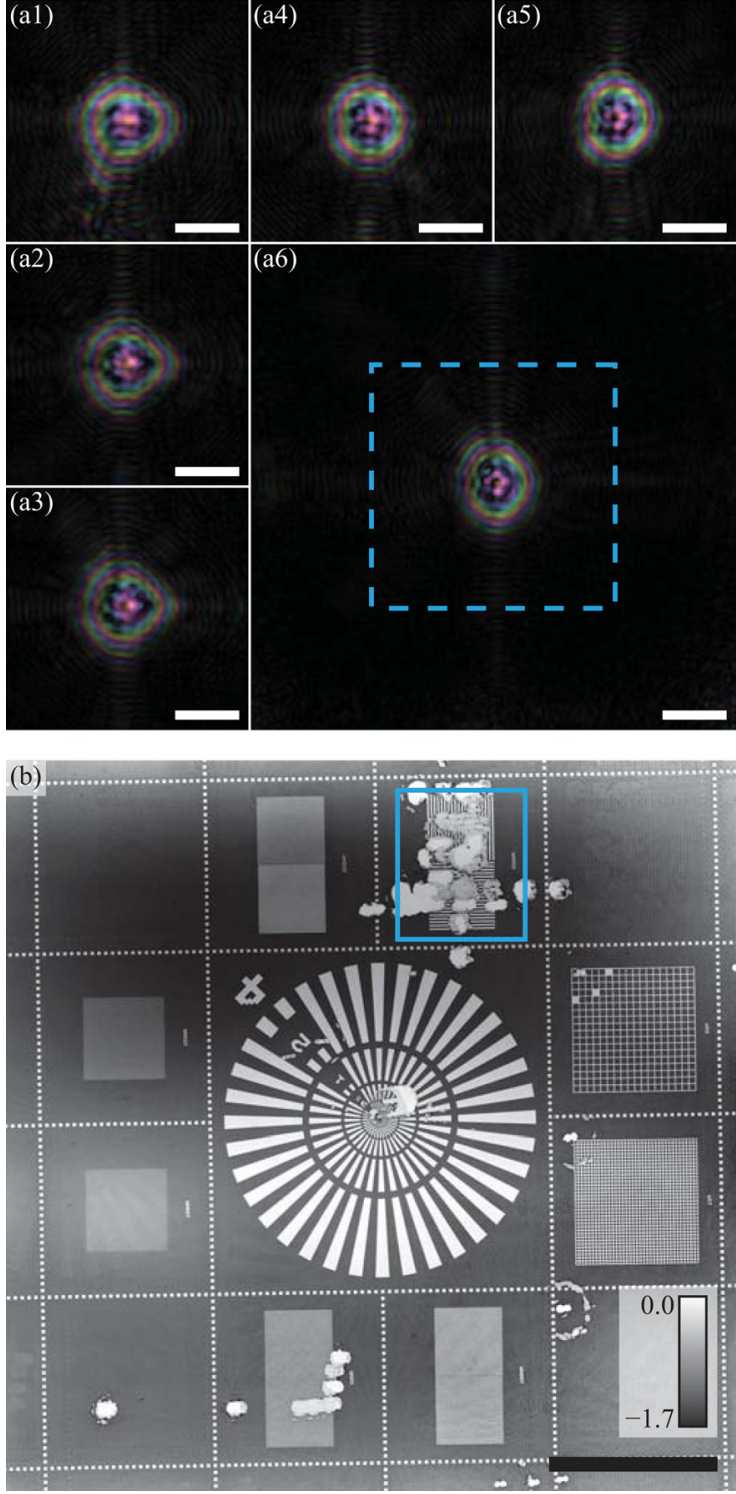

FIG. 3. Reconstruction with 6 beams. (a1)-(a6) The six reconstructed probes, with the arrangement according to the corresponding pinholes. The white scale bars represent $5 \mu \mathrm{m}$. Only (a6) shows the full probe, (a1)-(a5) show only the central part. Their field of view is indicated by the blue dashed square in (a6). (b) The reconstructed phase shift of the Siemens star. The area indicated by the blue rectangle is shown enlarged in Fig. 4. The black scale bar represents $50 \mu \mathrm{m}$.

deviations to smaller beam separations, centered around $46 \mu \mathrm{m}$, and we reconstruct the probes with this separation (accordingly $92 \mu \mathrm{m}$ for the isolated sixth beam). Consequently, the area that was scanned by the beams shrinks slightly to $212 \mu \mathrm{m} \times 212 \mu \mathrm{m}$. The six reconstructed beams are shown in Figs. 3(a1)-3(a6) and are arranged in the same order as they illuminate the sample [see Fig. 1(c)]. The reconstructed object is shown in Fig. 3(b) with the Siemens star in the center, structures of lines and spaces above and below it, and squares of different sizes to the left and right. In a previous experiment, the tantalum
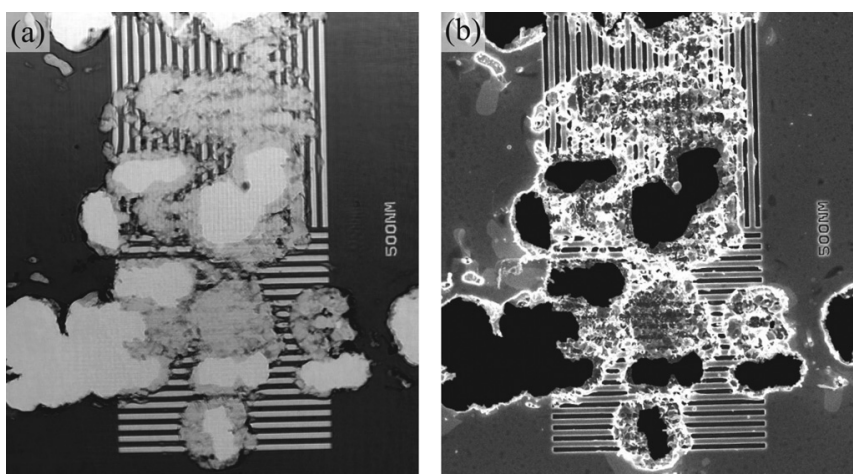

FIG. 4. Comparison of the damaged $500 \mathrm{~nm}$ lines and spaces on the Siemens star sample: (a) the six-beam reconstruction and (b) SEM image of the same area.

structures on the sample were in parts destroyed, creating the irregular features. The most damaged area is on the top right, indicated by the blue square in Fig. 3(b). This detail is shown enlarged in Fig. 4, illustrating the reconstruction quality.

Scanning this area with the same fluence using just a single beam would have taken more than thrice as long. Ideally, the six beams could achieve a speedup as high as six. Here, the overlap between the individual scan areas reduces the speedup. Scans with a smaller overlap are difficult to reconstruct because the high similarity between the six beams makes it difficult for the reconstruction to correctly separate the individual contributions of each beam. With little or no overlap, the reconstruction algorithm has no direct way to discern, which beam creates a certain diffraction feature. To achieve the full speedup in future experiments, the beams should be individualized.

In conclusion, we have demonstrated how $3 \mathrm{D}$ nanoprinting offers a more adaptable way to construct x-ray multi-beam optics. By using this technology, we could create a dedicated multi-beam setup with a flexible beam arrangement. As refractive x-ray lenses can be placed closer to each other than previous multi-beam optics, they are better suited to utilize more of the incoming photon flux. Using six beams with a maximum beam separation of $92 \mu \mathrm{m}$, we could image an area of $212 \mu \mathrm{m} \times 212 \mu \mathrm{m}$, more than three times larger than the mechanical scan area of $120 \mu \mathrm{m} \times 120 \mu \mathrm{m}$. It is still open how high the number of simultaneous beams can be pushed, leaving the potential for even higher scan speedups. This would allow us to image samples of millimeter size for needle-in-the-haystack problems in a useful time span. Potential limiting criteria can be the size of the flat beam, the lifetime of the lenses, ${ }^{25}$ or the count rate of the detector.

Currently, the high similarity between the different beams is limiting the speedup and has proven to be a challenge for the reconstruction. Due to the highly reproducible printing process, the lens towers and their respective probes are essentially clones, and the only differences are introduced due to small manufacturing errors. Disentangling the diffraction from many nearly identical beams in the reconstruction requires high iteration numbers. To allow larger beam numbers in the future, this can be prevented by making the probes more unique, ideally even mutually orthogonal, for example, by adding a particular orbital angular momentum to each beam. 
We acknowledge DESY (Hamburg, Germany), a member of the Helmholtz Association HGF, for the provision of experimental facilities. Parts of this research were carried out at PETRA III within experiment 11005480, and we would like to thank Gerald Falkenberg for assistance in using the beamline P06. This research was supported in part through the Maxwell computational resources operated at DESY and by core funding of the Helmholtz Imaging Platform (HIP), a platform of the Helmholtz Incubator on Information and Data Science. M.S. acknowledges funding from the Deutsche Forschungsgemeinschaft (DFG)-SFB 1441-Project-ID 426888090 .

\section{DATA AVAILABILITY}

The data that support the findings of this study are available from the corresponding author upon reasonable request.

\section{REFERENCES}

1F. Pfeiffer, "X-ray ptychography," Nat. Photonics 12, 9-17 (2018).

${ }^{2}$ K. Stachnik, M. Warmer, I. Mohacsi, V. Hennicke, P. Fischer, J. Meyer, T. Spitzbart, M. Barthelmess, J. Eich, C. David, C. Feldmann, B. Busse, K. Jähn, U. E. Schaible, and A. Meents, "Multimodal x-ray imaging of nanocontainertreated macrophages and calcium distribution in the perilacunar bone matrix," Sci. Rep. 10, 1784 (2020).

${ }^{3}$ J. Becher, T. L. Sheppard, Y. Fam, S. Baier, W. Wang, D. Wang, S. Kulkarni, T. F. Keller, M. Lyubomirskiy, D. Brueckner, M. Kahnt, A. Schropp, C. G. Schroer, and J.-D. Grunwaldt, "Mapping the pore architecture of structured catalyst monoliths from nanometer to centimeter scale with electron and x-ray tomographies," J. Phys. Chem. C 123, 25197-25208 (2019).

${ }^{4}$ M. Holler, M. Guizar-Sicairos, E. H. R. Tsai, R. Dinapoli, E. Müller, O. Bunk, J. Raabe, and G. Aeppli, "High-resolution non-destructive three-dimensional imaging of integrated circuits," Nature 543, 402-406 (2017).

${ }^{5}$ M. Guizar-Sicairos, I. Johnson, A. Diaz, M. Holler, P. Karvinen, H.-C. Stadler, R. Dinapoli, O. Bunk, and A. Menzel, "High-throughput ptychography using Eiger: Scanning x-ray nano-imaging of extended regions," Opt. Express 22, 14859-14870 (2014).

${ }^{6}$ IARPA, see https://www.iarpa.gov/index.php/research-programs/raven/ravenbaa for "Rapid Analysis of Various Emerging Nanoelectronics (RAVEN)" (2015).

${ }^{7}$ For $\mathrm{x}$-ray imaging, increasing the resolution fourfold requires a $4^{4}=256$ times stronger signal, either by increasing the flux or the exposure time.

${ }^{8}$ C. Bevis, R. Karl, Jr., J. Reichanadter, D. F. Gardner, C. Porter, E. Shanblatt, M. Tanksalvala, G. F. Mancini, H. Kapteyn, M. Murnane, and D. Adams, "Multiple beam ptychography for large field-of-view, high throughput, quantitative phase contrast imaging," Ultramicroscopy 184, 164-171 (2018).

${ }^{9} \mathrm{M}$. Hirose, T. Higashino, N. Ishiguro, and Y. Takahashi, "Multibeam ptychography with synchrotron hard x-rays," Opt. Express 28, 1216-1224 (2020).

${ }^{10}$ Y. Yao, Y. Jiang, J. A. Klug, M. Wojcik, E. R. Maxey, N. S. Sirica, C. Roehrig, Z. Cai, S. Vogt, B. Lai, and J. Deng, "Multi-beam x-ray ptychography for highthroughput coherent diffraction imaging," Sci. Rep. 10, 19550 (2020).

${ }^{11}$ M. Malinauskas, M. Farsari, A. Piskarskas, and S. Juodkazis, "Ultrafast laser nanostructuring of photopolymers: A decade of advances," Phys. Rep. 533, 1-31 (2013).
${ }^{12}$ U. T. Sanli, H. Ceylan, I. Bykova, M. Weigand, M. Sitti, G. Schütz, and K. Keskinbora, “3D nanoprinted plastic Kinoform x-ray optics," Adv. Mater. 30, 1802503 (2018).

${ }^{13}$ A. K. Petrov, V. O. Bessonov, K. A. Abrashitova, N. G. Kokareva, K. R. Safronov, A. A. Barannikov, P. A. Ershov, N. B. Klimova, I. I. Lyatun, V. A. Yunkin, M. Polikarpov, I. Snigireva, A. A. Fedyanin, and A. Snigirev, "Polymer $\mathrm{X}$-ray refractive nano-lenses fabricated by additive technology," Opt. Express 25, 14173-14181 (2017).

${ }^{14}$ M. Lyubomirskiy, F. Koch, K. A. Abrashitova, V. O. Bessonov, N. Kokareva, A. Petrov, F. Seiboth, F. Wittwer, M. Kahnt, M. Seyrich, A. A. Fedyanin, C. David, and C. G. Schroer, "Ptychographic characterisation of polymer compound refractive lenses manufactured by additive technology," Opt. Express 27, 8639-8650 (2019).

${ }^{15}$ C. G. Schroer, M. Seyrich, A. Schropp, R. Döhrmann, S. Botta, P. Wiljes, D. Brückner, M. Kahnt, F. Wittwer, L. Grote, D. Koziej, J. Garrevoet, and G. Falkenberg, "Ptychographic Nano-Analytical microscope (PtyNAMi) at PETRA III: Signal-to-background optimization for imaging with high sensitivity," Proc. SPIE 11112, 111120D (2019).

${ }^{16}$ I. Johnson, A. Bergamaschi, H. Billich, S. Cartier, R. Dinapoli, D. Greiffenberg, M. Guizar-Sicairos, B. Henrich, J. Jungmann, D. Mezza, A. Mozzanica, B. Schmitt, X. Shi, and G. Tinti, "Eiger: A single-photon counting X-ray detector," J. Instrum. 9, C05032 (2014).

${ }^{17}$ J. Reinhardt, R. Hoppe, G. Hofmann, C. D. Damsgaard, J. Patommel, C. Baumbach, S. Baier, A. Rochet, J.-D. Grunwaldt, G. Falkenberg, and C. G. Schroer, "Beamstop-based low-background ptychography to image weakly scattering objects," Ultramicroscopy 173, 52-57 (2017).

${ }^{18}$ P. Thibault, M. Dierolf, O. Bunk, A. Menzel, and F. Pfeiffer, "Probe retrieval in ptychographic coherent diffractive imaging," Ultramicroscopy 109, 338-343 (2009).

${ }^{19}$ P. Thibault, M. Dierolf, A. Menzel, O. Bunk, C. David, and F. Pfeiffer, "Highresolution scanning $\mathrm{x}$-ray diffraction microscopy," Science 321, 379-382 (2008).

${ }^{20}$ A. Schropp, R. Hoppe, V. Meier, J. Patommel, F. Seiboth, H. J. Lee, B. Nagler, E. C. Galtier, B. Arnold, U. Zastrau, J. B. Hastings, D. Nilsson, F. Uhlén, U. Vogt, H. M. Hertz, and C. G. Schroer, "Full spatial characterization of a nanofocused $\mathrm{x}$-ray free-electron laser beam by ptychographic imaging," Sci. Rep. 3, 1633 (2013).

${ }^{21}$ D. J. Batey, D. Claus, and J. M. Rodenburg, "Information multiplexing in ptychography," Ultramicroscopy 138, 13-21 (2014).

${ }^{22}$ M. Guizar-Sicairos, S. T. Thurman, and J. R. Fienup, "Efficient subpixel image registration algorithms," Opt. Lett. 33, 156-158 (2008).

${ }^{23}$ D. J. Batey, T. B. Edo, C. Rau, U. Wagner, Z. D. Pešić, T. A. Waigh, and J. M. Rodenburg, "Reciprocal-space up-sampling from real-space oversampling in $\mathrm{x}$ ray ptychography,” Phys. Rev. A 89, 043812 (2014).

${ }^{24}$ F. Wittwer, R. Hoppe, F. Seiboth, J. Reinhardt, M. Scholz, and C. G. Schroer, "Ptychography with a virtually enlarged illumination," Microsc. Microanal. 24, 48-49 (2018).

${ }^{25}$ A. Barannikov, M. Polikarpov, P. Ershov, V. Bessonov, K. Abrashitova, I. Snigireva, V. Yunkin, G. Bourenkov, T. Schneider, A. A. Fedyanin, and A. Snigirev, "Optical performance and radiation stability of polymer x-ray refractive nano-lenses," J. Synchrotron Radiat. 26, 714-719 (2019).

${ }^{26}$ F. Seiboth, M. Kahnt, M. Lyubomirskiy, M. Seyrich, F. Wittwer, T. Ullsperger, S. Nolte, D. Batey, C. Rau, and C. G. Schroer, "Refractive hard X-ray vortex phase plates," Opt. Lett. 44, 4622-4625 (2019). 